British Journal of Nutrition (2021), 125, 618-627

doi: $10.1017 /$ S0007114520003128

(C) The Author(s), 2020. Published by Cambridge University Press on behalf of the Nutrition Society. This is an Open Access article, distributed under the terms of the Creative Commons Attribution licence (http://creativecommons.org/licenses/by/4.0/), which permits unrestricted re-use, distribution, and reproduction in any medium, provided the original work is properly cited.

\title{
Selenium and viral infection: are there lessons for COVID-19?
}

\author{
Giovanna Bermano ${ }^{1 *} \dagger$, Catherine Méplan ${ }^{2 *}+$, Derry K. Mercer ${ }^{1}$ and John E. Hesketh ${ }^{1}{ }^{*} \dagger$ \\ ${ }^{1}$ Centre for Obesity Research and Education (CORE), School of Pharmacy and Life Sciences, Robert Gordon University, Aberdeen \\ AB10 7GJ, UK \\ ${ }^{2}$ School of Biomedical, Nutritional and Sport Sciences, Faculty of Medical Sciences, Newcastle University, Newcastle upon Tyne \\ NE2 $4 H H, U K$
}

(Submitted 17 June 2020 - Final revision received 22 July 2020 - Accepted 23 July 2020 - First published online 6 August 2020)

\section{Abstract}

Se is a micronutrient essential for human health. Sub-optimal Se status is common, occurring in a significant proportion of the population across the world including parts of Europe and China. Human and animal studies have shown that Se status is a key determinant of the host response to viral infections. In this review, we address the question whether Se intake is a factor in determining the severity of response to coronavirus disease 2019 (COVID-19). Emphasis is placed on epidemiological and animal studies which suggest that Se affects host response to RNA viruses and on the molecular mechanisms by which Se and selenoproteins modulate the inter-linked redox homeostasis, stress response and inflammatory response. Together these studies indicate that Se status is an important factor in determining the host response to viral infections. Therefore, we conclude that Se status is likely to influence human response to the severe acute respiratory syndrome coronavirus 2 (SARS$\mathrm{CoV}-2$ ) infection and that Se status is one (of several) risk factors which may impact on the outcome of SARS-CoV-2 infection, particularly in populations where Se intake is sub-optimal or low. We suggest the use of appropriate markers to assess the Se status of COVID-19 patients and possible supplementation may be beneficial in limiting the severity of symptoms, especially in countries where Se status is regarded as suboptimal.

Key words: Selenium: COVID-19: Inflammation: Viral infection: Severe acute respiratory syndrome coronavirus 2: Redox status: Micronutrients

A link between Se deficiency and human disease was first demonstrated by the discovery in China that the aetiology of Keshan disease involved both coxsackievirus infection and a low intake of the micronutrient $\mathrm{Se}^{(1,2)}$. Since those initial observations, several studies have investigated how Se intake affects responses to viral infections ${ }^{(3)}$ and the importance of understanding these links is highlighted by the coronavirus disease 2019 (COVID19) pandemic $^{(4)}$. This is of particular relevance because, although severe Se deficiency is rare, there is considerable evidence that sub-optimal Se status is common with a significant proportion of the population across the world potentially in this category ${ }^{(5)}$. Fish and shellfish are relatively high in Se content but, for most foods, Se content is dependent on the level of Se present in the soil; this geographical variation is reflected in the Se status of different human populations, many of which have blood Se that is regarded as sub-optimal $(\leq 85 \mu \mathrm{g} / \mathrm{l})^{(5,6)}$, for example, in parts of Europe including the UK.

Severe acute respiratory syndrome coronavirus 2 (SARS$\mathrm{CoV}-2$ ) is the infectious agent responsible for the current COVID-19 pandemic. It is a coronavirus with a positive-sense single-stranded RNA genome (26-32 kb), one of the largest of the RNA viruses ${ }^{(7,8)}$. Other clinically important RNA viruses include those responsible for respiratory diseases, such as severe acute respiratory syndrome (SARS), Middle East respiratory syndrome (MERS) and influenza, and for other infectious diseases such as poliomyelitis and HIV infection ${ }^{(3)}$. SARS-CoV-2 targets cells expressing the angiotensin-converting enzyme 2 receptor; these include airway epithelial cells, alveolar epithelial cells, vascular endothelial cells and macrophages in the lung as well as myocardial and renal cells ${ }^{(9,10)}$. SARS-CoV-2 infection is

Abbreviations: COVID-19, coronavirus disease 2019; CVB, coxsackievirus B3; ER, endoplasmic reticulum; GPX, glutathione peroxidase; IFN, interferon; IV, influenza virus; MERS, Middle East respiratory syndrome; NF-kB, nuclear factor kappa-light-chain-enhancer of activated B cells; NOX, NADPH oxidase; Nrf2, nuclear factor erythroid 2-related factor 2; ROS, reactive oxygen species; SARS-CoV-2, severe acute respiratory syndrome coronavirus 2; SELENOF, selenoprotein F; SELENOS, selenoprotein S; TXNRD, thioredoxin reductase; UPR, unfolded protein response.

* Corresponding authors: Dr Giovanna Bermano, email g.bermano@rgu.ac.uk; Dr Catherine Méplan, email catherine.meplan@newcastle.ac.uk; Professor John E. Hesketh, email j.hesketh@rgu.ac.uk

$\dagger$ These authors contributed equally to this work. 
characterised by a highly aggressive inflammatory response. Pyroptosis of infected lung epithelial cells, as a result of viral replication, triggers an inflammatory cascade leading to the release of pro-inflammatory cytokines and chemokines ${ }^{(9)}$. The cytokine storm (hypercytokinaemia) observed in severe cases induces widespread damage to the lung epithelium that can facilitate the development of secondary bacterial or fungal infections ${ }^{(11)}$. In addition to the local damage, the cytokine storm and uncontrolled inflammation affect other parts of the body, in particular the myocardial and renal systems, and can lead to septic shock, multi-organ failure and death ${ }^{(12,13)}$.

The COVID-19 pandemic raises three major questions in relation to Se: (i) to what extent does Se intake, particularly sub-optimal intake as well as severe deficiency, affect responses to viral infection; (ii) what are the biochemical mechanisms supporting a role of Se in the host response and (iii) could Se intake be a factor in determining the response to COVID-19 infection? In this article, we address these questions by firstly reviewing the literature concerning the effects of Se on RNA virus infections, secondly by exploring how Se modulates the inter-linked redox homeostasis, stress response and inflammatory response mechanisms and finally by considering these effects in relation to recent observations on COVID-19.

\section{Host selenium status and viral infection}

The first evidence that there might be a link between Se status and susceptibility of humans to a viral infection came from the study of Keshan disease, a myocardiopathy associated with heart failure and death, among the population in Heilongjiang province in China ${ }^{(1)}$. The Se content of food and drinking water was found to be extremely low in areas where the disease was endemic. Addition of Se-containing fertiliser to the soil and direct nutritional supplementation led to a large reduction in the incidence of the acute form of the disease ${ }^{(1,14)}$. However, the seasonal variation of the disease suggested additional contributing factors, and postmortem samples from patients were found to contain the coxsackievirus B3 (CVB) virus, a single-stranded RNA virus. It is now clear that Keshan disease is due to a combination of infection with CVB and low Se status. In mice, CVB can lead to myocarditis, inflammation of the heart. The virulence of CVB strains varies and crucially it was observed that a non-virulent strain, which caused no disease in animals fed a Se-adequate diet, caused severe myocarditis in animals fed a Se-deficient diet ${ }^{(14)}$. In addition, sequencing of the viral genome revealed that passage through Se-deficient mice led to mutations in the viral genetic sequence and increased pathogenicity ${ }^{(15,16)}$. Similarly, an influenza virus (IV) strain causing mild pneumonitis induced much greater pathogenicity in Se-deficient mice ${ }^{(17,18)}$. Furthermore, as with CVB, Se status also affected IV mutation, with low Se status promoting rapid evolution in haemagglutinin $(H A)$ and neuraminidase $(N A)$ genes $^{(19)}$. An in vitro study on bronchial epithelial cells showed that Se supplementation lowered the extent of apoptotic cell death after infection with $\mathrm{IV}^{(18)}$.

Few data are available concerning the relationship between Se status and HIV susceptibility or disease progression ${ }^{(3)}$.
However, two studies indicate that sub-optimal Se status lowers the number of CD4 T cells and increases both disease progression and death rates in HIV-infected patients ${ }^{(20,21)}$. Se deficiency is commonly observed in HIV-infected patients and has been linked to increased mortality. In a study of 125 HIV-1 seropositive drug-using men and women, CD 4 T cell counts over time and Se deficiency (but not deficiency of vitamin A, vitamin $\mathrm{B}_{12}$ or $\mathrm{Zn}$ ) were significantly associated with mortality, indicating that Se deficiency is an independent predictor of survival for those with HIV-1 infection ${ }^{(21)}$.

The incidence of haemorrhagic fever with renal syndrome, a public health problem in China caused by RNA hantaviruses, is about six times higher in severely Se-deficient areas of China compared with non-Se-deficient areas, and negatively correlated with the Se content of crops and feeds ${ }^{(22)}$. Surprisingly, plasma concentration and activity of the selenoprotein glutathione peroxidase 3 (GPX3) were significantly higher in haemorrhagic fever with renal syndrome patients compared with controls, but this may indicate increased inflammation rather than differences in Se status. Finally, in vitro Se supplementation $(200 \mathrm{ng} / \mathrm{ml})$ significantly lowered viral copy number in human umbilical vein endothelial cells (HUVEC) infected with hantavirus at a low multiplicity of infection ${ }^{(22)}$. Although no human studies have assessed the impact of Se status on the disease associated with West Nile virus, another RNA virus, in vitro infection of kidney epithelial cells leads to lower virus-induced cell death in Se-supplemented conditions ${ }^{(23)}$.

The effects of Se supplementation on viral infection in humans were studied in UK volunteers of marginal Se status (plasma Se $<95 \mu \mathrm{g} / \mathrm{l}$ ) who were given an attenuated polio virus. Se supplementation was found to both increase the rate at which the virus was cleared from the body and decrease the rate at which mutations appeared in the viral genome ${ }^{(24)}$. In addition, measurements of immune markers indicated that supplemental Se accelerated the cellular antiviral response. Importantly, this study not only supports animal work on IV and CVB showing that higher Se status enhanced the host response to a viral infection and promoted generation of mutations that increase virulence of RNA viruses, but also provides evidence that similar effects occur in humans within a range of Se status found commonly in the UK population and worldwide.

In summary, there is strong evidence that low Se status in both animals and humans influences the host response to a number of RNA viruses with low Se status leading to more severe forms of the disease. Furthermore, in CVB and IV infections, low Se status also promotes generation of mutations and increased virulence. The impact of Se status on the ability of a host to respond to viral infection is reflected in the crucial role of Se and selenoproteins in cellular and molecular mechanisms involved in the control of redox homeostasis, stress response, immune and inflammatory response.

\section{Selenoproteins, cell stress response mechanisms and viral infection}

Dietary Se is converted in the body into the amino-acid selenocysteine which is then incorporated into proteins, 


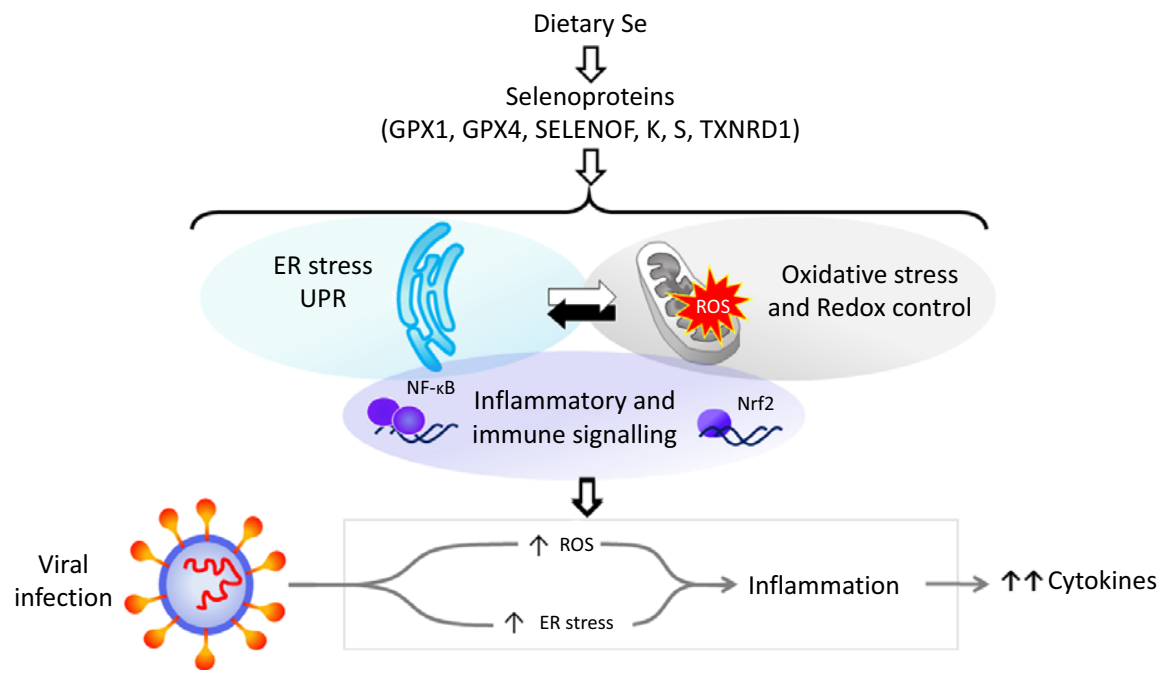

Fig. 1. Selenium and response to viral infection. In humans, dietary selenium is incorporated into twenty-five selenoproteins as the amino acid selenocysteine. Selenoproteins include glutathione peroxidases (GPX), $15 \mathrm{kDa}$ selenoprotein F (SELENOF), selenoproteins $\mathrm{K}$ and $\mathrm{S}$ and thioredoxin reductases (TXNRD). They play crucial roles in molecular pathways such as oxidative stress response, redox control and mitochondrial function, as well as endoplasmic reticulum (ER) stress and unfolded protein responses (UPR), and immune and inflammatory response in particular involving NF- $\kappa$ B and nuclear factor erythroid 2-related factor 2 (Nrf2) signalling. Cross-talk between these pathways has been shown to be key to produce an adequate response to viral infections, and low selenium status has been linked to poorer response to RNA virus infection in human studies, and animal and cell culture models. The diagram illustrates how reduced expression of selenoproteins as a result of low/sub-optimal selenium status could alter molecular pathways involved in stress responses and contribute to an aggressive pro-inflammatory environment leading to poorer disease prognosis.

selenoproteins, during their synthesis by a unique mechanism which involves a specific tRNA for selenocysteine and recoding of a UGA stop codon as codon for selenocysteine ${ }^{(25,26)}$. To date, twenty-five genes coding for selenoproteins have been identified. The biological activity of Se is carried out primarily through its presence in these selenoproteins, the functions of which centre around roles in antioxidant protection, redox homeostasis and endoplasmic reticulum (ER) stress ${ }^{(27)}$ (Fig. 1). Thus, GPX1-4 have well-documented, important roles in the metabolism of, and protection from, reactive oxygen species (ROS) including peroxides and lipid hydroperoxides. Thioredoxin reductases (TXNRD) function in redox homeostasis and signalling, and a series of less well-characterised selenoproteins including selenoprotein $\mathrm{F}, \mathrm{K}, \mathrm{M}, \mathrm{N}$ and S (SELENOF, SELENOK, SELENOM, SELENON and SELENOS) are present in the ER where they play roles in protein folding and protection from oxidative stress. In both human and mouse models, transcriptomic and proteomic analyses have indicated that, in colonic tissue and leucocytes, the major downstream target pathways affected by sub-optimal Se intake are immune response and inflammatory signalling, nuclear factor kappa-light-chain-enhancer of activated B cells $(\mathrm{NF}-\kappa \mathrm{B})$ signalling and ER stress response pathways ${ }^{(28-30)}$. Such changes are consistent with the known roles of selenoproteins and highlight that changes in Se intake can affect the pathways that determine cell responses to oxidative and ER stress as well as the immune response.

\section{Oxidative stress response and redox homeostasis}

Viral-induced oxidative stress through generation of ROS is crucial to the viral lifecycle and its pathogenicity ${ }^{(31)}$. Se, through the activity of antioxidant selenoproteins, has the potential to enhance the response to viral-induced oxidative stress and to contribute to the maintenance of redox homeostasis. Se supplementation, which increases GPX1 expression and lowers oxidative stress in a cell culture model, reduces the replication rate of porcine circovirus 2, a DNA virus ${ }^{(32)}$. Data from GM animal models (knock-out) show that, contrary to wild-type mice, animals lacking GPX1 developed myocarditis when infected by a benign CVB strain, in a similar manner to mice fed a Se-deficient diet ${ }^{(33)}$. Similarly, following IV infection, GPX1 knock-out mice displayed an increased and prolonged tissue inflammation, higher levels of macrophages in bronchial fluid and higher levels of the pro-inflammatory cytokine TNF- $\alpha$; furthermore, some effects were reversed by ebselen (2-phenyl-1,2-benzisoselenazol$3(2 \mathrm{H})$-one), a synthetic organoselenium compound/drug with GPX-mimetic properties ${ }^{(34)}$. Lower selenoprotein levels in mice expressing a mutant selenocysteine tRNA gene were found to alter the response to IV infection, with increased levels of lung pro-inflammatory cytokines and interferon- $\gamma$ (IFN- $\gamma$ ) and slow viral clearance rates, although there was no effect on lung pathology ${ }^{(35)}$. Since these mice showed only a small $20 \%$ reduction in GPX1 expression, it is possible that there is a threshold activity of GPX1 and other selenoproteins above which there is little effect on virus infection. Overall, these data suggest that GPX1 is important for appropriate cell and tissue responses to a viral infection, in particular in relation to the inflammatory response to respiratory viruses.

Changes in redox homeostasis, disruption of antioxidant defences, chronically elevated levels of ROS and induction of ROS-generating enzymes, such as NADPH oxidase (NOX) and xanthine oxidase, are key events linked to infection with respiratory viruses, including IV and SARS-CoV ${ }^{(31,36)}$. Increased levels of ROS and reactive nitrogen species have been reported in lung 
tissues of patients who died in fatal IV pandemics, while studies with IV-infected mice and cell lines also demonstrated an enhanced production of ROS and disturbance of antioxidant defence ${ }^{(37-39)}$. Suppression of chronic oxidative stress by targeting pro-oxidant enzymes such as NOX and dual oxidase (DUOX) could, therefore, ameliorate host response to viral infection. At present, no evidence is available for a role of Se or selenoproteins in modulating NOX and DUOX activity in relation to viral infections; however, supplementation with selenomethionine has been shown to protect lung tissue against toxic effects of ionising radiation by reducing DUOX 1 and 2 gene expression and IL- 4 and IL- 4 receptor subunit $\alpha-1$ protein levels ${ }^{(40)}$. Moreover, GPX and TXNRD have been shown, in endothelial cells, to play an important role in controlling the metabolism and physiological function of ROS and reactive nitrogen species, whose production is regulated by NOX and xanthine oxidase ${ }^{(41)}$. Control of ROS levels associated with endothelial damage and inflammation may provide a potential mechanism by which Se status modulates the host response to viral infection, and this could be significant in the case of SARS-CoV-2 infection as both endothelial damage and inflammation have been linked to COVID-19 aetiology ${ }^{(42)}$.

As a first line of defence, epithelial barriers often undergo significant damage as a result of the release/production of ROS. Through their antioxidant functions, selenoenzymes such as GPX protect epithelial barriers from ROS by reducing lipid and phospholipid hydroperoxides, and thus, adequate Se status could also contribute to the maintenance of the structural and functional integrity of respiratory epithelial barriers. Similarly, antioxidant selenoproteins protect neutrophils from endogenous oxidative stress ${ }^{(43)}$.

Mitochondrial dysfunction has also been linked to viral infection. In IV infection, mitochondria are an important source of ROS due to the virus-induced leakage of electrons from the respiratory chain ${ }^{(44)}$. Similarly, a study by Shi et al. ${ }^{(45)}$ reported that a protein encoded by SARS-CoV, designated as open reading frame-9b (ORF-9b), localised to mitochondria and caused mitochondrial elongation, resulting in evasion of host innate immunity. Although to date there is no evidence that during respiratory virus infection the alteration of mitochondrial function is caused by lower selenoprotein expression, a considerable amount of data indicates that selenoproteins such as GPX4, GPX1 and TXNRD1 are crucial to maintain mitochondrial function and redox homeostasis ${ }^{(46,47)}$. Thus, low Se status has the potential to affect response to respiratory viruses by impacting on the expression of selenoproteins key to mitochondrial function (Fig. 1).

\section{Endoplasmic reticulum stress}

The ER is important for protein synthesis, folding, processing and post-translational modifications. During viral infections, the protein load exceeds the ER folding and processing capacity, and activation of various signalling pathways, collectively known as ER stress response or unfolded protein response (UPR), occurs as part of the host antiviral response ${ }^{(48)}$. ER redox state, ER stress and $\mathrm{Ca}^{2+}$ signalling are tightly regulated by a complex antioxidant system, which includes seven ER-resident selenoproteins - $15 \mathrm{kDa}$ selenoprotein (SELENOF), type 2 iodothyronine deiodinase (DIO2) and SELENOS, N, K, M and T (Fig. 1). As a group, they are implicated in a range of processes including ER stress and inflammation (reviewed in Addinsall et $\left.a l .{ }^{(49)}\right)$. Cross-talks between ER function, NF- $\kappa \mathrm{B}$ signalling and mitochondrial function ${ }^{(50)}$ during immune response and antiviral defence highlight that these mechanisms are interlinked (Fig. 1). More specifically, in Z $\alpha$ (1)-antitrypsin (ZAAT) deficiency, a disease associated with emphysematous lung disease, SELENOS was found to affect ER function and NF- $\kappa \mathrm{B}$ signalling, and this effect was enhanced following Se supplementation $^{(51)}$. In addition, silencing of SELENOS in vascular smooth muscle cells, enhanced markers of ER stress suggesting that SELENOS might protect cells by inhibiting oxidative and ER stress $^{(52)}$. Mice with targeted deletion of SELENOK exhibited decreased viral clearance and elevated viral titres in the brain upon West Nile virus infection. Interestingly, these mice showed decreased $\mathrm{Ca}^{2+}$ flux in the ER of T cells, macrophages and neutrophils and lower $\mathrm{T}$ cell proliferation suggesting a role for SELENOK in immune response ${ }^{(53)}$. In human volunteers, SELENOS expression in peripheral blood mononuclear cells was found to increase after an IV challenge, and furthermore this increase was enhanced after Se supplementation ${ }^{(54)}$.

These findings, although not all closely related to respiratory viral infections, demonstrate several roles for selenoproteins in regulating ER stress and suggest they are important in determining ER stress and inflammatory responses during viral infections. SARS-CoV accessory viral protein affects several UPR components, suggesting that such viruses may directly modulate ER stress responses ${ }^{(55)}$. Since ER stress and inflammation are inter-linked $^{(56)}$, it is likely that UPR induction may play a significant role in the host response to SARS-CoV-2. Furthermore, there is a close relationship between ER stress, oxidative stress and inflammation. ER stress can lead to inflammation and conversely inflammation can induce UPR and ER stress ${ }^{(56)}$. High production of free radicals by immune cells, especially macrophages, at the site of infection, triggers oxidative stress. Excessive extracellular ROS/reactive nitrogen species initiates signalling cascades that lead to the onset of the inflammatory response, generating inflammatory mediators such as IL- $1 \beta$, IL- 6 and TNF- $\alpha$ and activating NF- $\kappa$ B signalling pathways ${ }^{(57-60)}$.

\section{Selenium, inflammation, immune response and viral infections}

As described in the above sections, there is extensive evidence suggesting that the inter-linked biochemical pathways of oxidative stress, ER stress and inflammation are induced during viral infection. This is true for a number of respiratory viruses and the limited data concerning SARS-CoV and SARS-COV-2 indicate that they affect these pathways. Lung cell lines infected with either SARS-CoV-2, IV or MERS-CoV showed that up-regulation of antiviral IFN signalling was observed with all three viral infections, whereas up-regulation of the cytokine/inflammatory processes, down-regulation of mitochondrial organisation and respiration processes, and perturbation in the autophagic processes were specifically observed in SARS-CoV-2-infected cells 
and were absent in IV-infected cells ${ }^{(61)}$. These data highlight that, in SARS-CoV-2 infection, both mitochondrial function and inflammatory signalling were affected, which in turn could affect the immune response and lead to severe outcomes in the host.

In infections caused by SARS-CoV or SARS-CoV-2, pronounced inflammation and increased secretion of IL- $1 \beta$, IL- 4 , IL-10, IFN- $\gamma$, IFN- $\gamma$-induced protein 10 (IP-10) and monocyte chemoattractant protein 1 (MCP-1) were observed ${ }^{(4,62)}$. In addition, production of several NF- $\kappa$ B-mediated cytokines, including IL-6 and IL-8, has been detected in human bronchial epithelial cells in response to SARS-CoV infection ${ }^{(63)}$, whereas patients with severe COVID-19 in intensive care units exhibit elevated plasma levels of several cytokines (IL-2, IL-7, IL-10, TNF- $\alpha$, MCP-1, granulocyte-colony stimulating factor (GCSF), IP-10 and macrophage inflammatory proteins (MIP1A)), suggesting a cytokine storm, resulting in hyper-inflammation ${ }^{(64)}$. Furthermore, evidence exists for IV strains to activate the nuclear factor erythroid 2-related factor 2/antioxidant response element (Nrf2/ARE) defence pathway, in vitro and in mice, subsequently leading to the activation of the NF- $\kappa \mathrm{B}$ pathway and cytokine production $^{(65,66)}$, suggesting a potential important role for the Nrf2NF- $\kappa$ B cross-talk in associated pathogenesis, even if further evidence is required for other respiratory viruses.

Moderate Se deficiency was found to activate Nrf2 and the Wnt pathways which may have negative effects on protection against oxidative stress and inflammation in cancer cell models $^{(67)}$; in addition, TXNRD1 has been shown to be a potent regulator of $\mathrm{Nrf} 2$ activation in lung epithelial cells ${ }^{(68)}$. Using a combined transcriptomics and proteomics approach to analyse gene and protein expression in rectal biopsies of healthy individuals, sub-optimal Se status was found to be associated with inhibition of NF- $\kappa \mathrm{B}$, IL- $1 \beta$, IL- 6 and TNF- $\alpha$ signalling and down-regulation of immune and inflammatory response pathways ${ }^{(30)}$. Similar effects were observed in the colon of mice fed a mildly deficient Se diet ${ }^{(28)}$. In mice, a genomic approach combined with cytokine measurements has shown that a low Se diet led to increased activity of IFN- $\gamma$ and IL- 6 pathways as well as IL-6 levels ${ }^{(69)}$. Furthermore, following a lipopolysaccharide challenge, IL-6 levels were found to be greater in mice fed a low Se diet ${ }^{(70)}$, while, in women, lower Se status was associated with higher plasma IL-6 levels ${ }^{(71)}$. In addition, uncontrolled inflammation is associated with an altered balance of pro-inflammatory and anti-inflammatory eicosanoids. Animal studies have shown that increased Se intake leads to a rebalance towards more anti-inflammatory eicosanoids ${ }^{(72)}$, probably through modulation of lipoxygenase and cyclooxygenase activities as a result of increased GPX activity lowering levels of hydroperoxides and lipid radicals and so altering peroxide tone. Increased Se intake also causes lower expression of pro-inflammatory $\mathrm{NF}-\kappa \mathrm{B}$ signalling ${ }^{(73)}$. There is also evidence for epigenetic regulation of inflammatory gene expression by $\mathrm{Se}^{(74)}$. Overall, Se intake affects a range of inflammatory mechanisms including Nrf2, eicosanoid and NF- $\kappa$ B signalling pathways $(30,67,68,72,73)$ and therefore, individuals with sub-optimal/low Se status may exhibit an inadequate inflammatory response to respiratory RNA viral infections.

Alongside inflammation, SARS-CoV-2 infection triggers a local immune response, recruiting macrophages and monocytes that respond to the infection, release cytokines and prime adaptive $\mathrm{T}$ and $\mathrm{B}$ cell immune responses ${ }^{(9)}$. In particular, the immune response induced by SARS-CoV-2 infection is two phased: an initial phase in which specific adaptive immune responses are required to eliminate the virus and to preclude disease progression to the second and more severe phase that is characterised by severe lung damage (e.g. acute respiratory distress syndrome) and in which suppression of inflammation may be beneficial ${ }^{(75)}$. Therefore, strategies to boost immune responses are certainly important as, in some cases, a dysfunctional immune response occurs, which can cause severe lung and even systemic pathology.

Adequate Se levels have been shown to be required for the differentiation and proliferation of a number of immune cells involved in innate and adaptive immunity. In particular, at moderate doses, Se supplementation increases $\mathrm{T}$ cell proliferation and natural killer cell activity ${ }^{(76,77)}$. Moreover, Se supplementation of individuals with marginally low plasma levels increased cellular immune response to live polio vaccine and virus clearance ${ }^{(24)}$; although Se supplementation did not directly affect antibody titre after flu vaccination ${ }^{(78)}$, supplementation of individuals with marginally low Se status $(92-98 \mu \mathrm{g} / \mathrm{l})$ resulted in some beneficial effect on cellular immunity, with significantly higher $\mathrm{T}$ cell proliferation in the group supplemented with $100 \mu \mathrm{g} / \mathrm{d}$ selenomethionine in yeast matrix ${ }^{(78)}$. However, higher supplementation doses $(200 \mu \mathrm{g} / \mathrm{d}$ selenomethionine in yeast matrix) led to a reduction in granzyme positive CD8 cells, and other forms of Se (e.g. Se-onions) did not impact on the immune response to flu vaccination ${ }^{(78)}$. These data complement the review by Steinbrenner and colleagues ${ }^{(79)}$ and suggest that optimising plasma Se status may improve immune responses, but high supplemental doses may have detrimental effects. Finally, as part of the innate immune response to pathogens, the methionine sulfoxide reductase B1 (MSRB1) selenoenzyme has been found, in murine macrophages, to regulate, through a redox mechanism and remodelling of the actin cytoskeleton, macrophage activation and phagocytosis ${ }^{(80)}$ and to promote anti-inflammatory cytokine production; the ablation of the $M S R B 1$ gene induced instead excessive pro-inflammatory cytokine production $^{(81)}$

\section{Selenium status and COVID-19}

The available data suggest that several respiratory viruses induce changes in redox, ER stress and inflammation pathways that may be modulated by Se or selenoproteins. It appears that SARS-CoV2 may induce comparable changes in these pathways but, to date, it is not known whether low Se status modulates the effect of the virus on such pathways and so enhances the cytokine storm observed in severe cases of COVID-19. Future studies should address whether this is the case, using both relevant animal and cell models and by assessing Se status and plasma inflammatory markers of patients with severe $v$. mild forms. Blood levels of SELENOP, the major form of Se in blood, are lowered by inflammation making the use of appropriate markers of Se status such as hair Se or erythrocyte Se important in human studies ${ }^{(82,83)}$. Such studies could inform the potential use of Se 


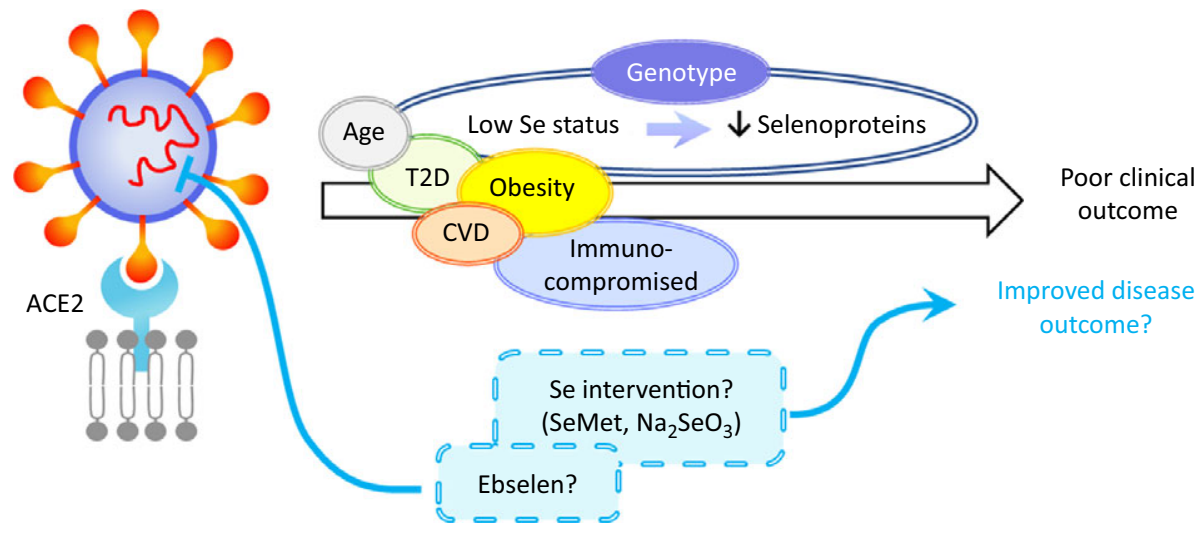

Fig. 2. Hypothetical model of the impact of selenium on severe acute respiratory syndrome coronavirus 2 (SARS-CoV-2) infection. Disease outcome of a coronavirus disease 2019 (COVID-19) patient is influenced by age, obesity and the presence of co-morbidities such as type 2 diabetes (T2D), CVD and by being immunocompromised. Based on the current knowledge of the effects of selenium status on RNA virus infections, it can be speculated that patients with low/sub-optimal selenium status and reduced selenoprotein expression could be at higher risk of poor disease outcome. This could be influenced not only by dietary intake but also by genotype for a number of genetic variants in selenoprotein genes. Nutritional intervention such as selenium supplementation with selenomethionine (SeMet) or sodium selenite $\left(\mathrm{Na}_{2} \mathrm{SeO}_{3}\right)$ may therefore improve disease outcomes for individuals with sub-optimal selenium status. Furthermore, therapeutic interventions may also include synthetic selenium compounds such as ebselen that has been found to inhibit SARS-CoV-2 main protease (Mro). ACE2, angiotensin-converting enzyme 2.

supplementation to limit the inflammatory response of patients (Fig. 2).

It is widely accepted that SARS-CoV-2 originated in Wuhan, Hubei province in China and rapidly spread across Hubei province and, subsequently, globally. Prior to the emergence of SARS-CoV-2, the population of Hubei was reported to show a wide range of hair Se content $(0 \cdot 06-0 \cdot 79 \mathrm{mg} / \mathrm{kg})$, albeit with a small sample size ${ }^{(84)}$. This wide range is compatible with both Se deficiency perhaps not being uncommon in Hubei province and there also being known high-Se areas. Interestingly, a recent ecological analysis using these data in relation to the COVID-19 outbreak in China indicates that there may be a relationship between Se status and disease outcome ${ }^{(85)}$. Cure and death rates from COVID-19 were analysed in different regions in relation to Se status as previously assessed by hair Se; notably, the city of Enshi in Hubei province, known for high Se status, showed a significantly higher cure rate, whereas Heilongjiang province, known to be an area of low Se status, showed a higher death rate. Overall, cure rate was associated with higher Se status. Although the association described in the present study is not based directly on Se status in COVID-19 patients, the work highlights that further, more detailed studies of Se status, COVID-19 infection and disease outcomes are vitally important. In addition, since levels of Se reported in some of the areas relevant to the present study are higher than those needed to optimise selenoprotein activities such future studies should address the range of Se intake effective at giving any protection.

Worst disease outcomes and higher mortality for COVID-19 patients have been associated with age, as well as other morbidities including obesity, type 2 diabetes and cardiovascular disorders that are often characterised by malnutrition ${ }^{(86)}$ and immunecompromised individuals (Fig. 2). Low levels, or intakes, of micronutrients including Se, $\mathrm{Zn}$ and vitamins $\mathrm{A}, \mathrm{B}_{6}, \mathrm{~B}_{12}$ and $\mathrm{E}$ have been linked to adverse outcomes in viral infection ${ }^{(87)}$ and levels of these micronutrients, as well as vitamins $\mathrm{C}$ and $\mathrm{D}, n-3$ PUFA and iron should be considered in COVID-19 patients ${ }^{(88)}$. Obesity and high BMI have been found to be associated with severe forms of COVID-19 ${ }^{(89,90)}$, possibly due to obesity-induced chronic inflammation. Potential explanations for these findings could include poor nutritional status of obese individuals, pre-existing impaired immune response or amplified pro-inflammatory responses and/or high affinity of SARSCoV-2 for angiotensin-converting enzyme 2 (ACE2) receptor, highly expressed in adipose tissue ${ }^{(91,92)}$. Similarly, obesity has been shown to increase the duration of IV shedding and to be an independent risk factor for hospitalisation and death in H1N1 influenza $^{(93,94)}$, and it has been suggested that the adipose tissue could represent a reservoir for SARS-CoV-2, IV and HIV ${ }^{(89,95)}$.

Furthermore, several studies indicate that Se levels are lower in obese individuals and a recent systematic review confirmed both that mean levels of Se in blood/serum were lower in the obese compared with healthy adult subjects and that mean blood GPX activity was decreased in obese individuals ${ }^{(96)}$. Interestingly, the authors reported that Se levels were higher in subjects with the metabolic syndrome.

Se derivative compounds have also been used as therapeutic agents. In particular, the organoselenium compound ebselen exhibits antioxidant properties, as well as anti-inflammatory, and anti-bacterial and antiviral properties ${ }^{(97)}$. Recently, using a combination of virtual and high-throughput technologies to screen 10000 compounds, ebselen was identified among the most efficient potential inhibitor against COVID-19 main protease $\left(\mathrm{M}^{\mathrm{pro}}\right)$ and demonstrated strong antiviral activity in cell-based assays ${ }^{(98,99)}$. As clinical trials have shown ebselen can be safely used in humans ${ }^{(100,101)}$, this compound represents an interesting potential therapeutic candidate for COVID-19 (Fig. 2).

Given the strong evidence that Se protects against viral infection and supports an adequate immune response, it seems that individuals with low Se status could benefit from supplemental Se to prevent the development of severe forms of COVID-19. However, considering the ongoing debate around the riskbenefit window at an individual level ${ }^{(26)}$, Se supplementation 
should be restricted to individuals with low or sub-optimal Se intake/status to avoid toxicity associated with high Se intake. Since a number of SNP in genes encoding selenoproteins have been found to have functional consequences for Se metabolism and risk for a number of multifactorial diseases ${ }^{(26)}$, it is possible that genetic factors may also influence any effect of Se status on viral infection progression. As regards selenoproteins' importance in redox homeostasis or ER stress response, a variant in the SELENOS gene has been reported to affect levels of inflammatory cytokines $^{(102)}$, one in GPX4 to affect monocyte adhesion to endothelial cells ${ }^{(103)}$ and others to affect inflammatory disease and cancer risk. It is interesting to speculate that such genetic variations, in combination with Se status, may impact on the ability of an individual to produce an adequate immune response to, and combat oxidative stress that occurs during viral infection (Fig. 2). Although to date no SNP in a Se pathway have been linked to altered response to infectious pathogens, other genetic variants in key host immune response genes in the host immune system have been shown to modulate both disease outcome and response to vaccination ${ }^{(104)}$.

An additional link, albeit novel and speculative, between SARS-CoV-2 and Se metabolism is through the mechanism of nonsense-mediated RNA decay. Coronaviruses, of which SARS-CoV-2 is one, have been reported to interfere with nonsense-mediated RNA decay ${ }^{(105)}$, a cellular mechanism that is also thought to play a role in regulating the pattern of synthesis of selenoproteins ${ }^{(106,107)}$. It can, therefore, be envisaged that SARS-CoV-2 infection may alter selenoprotein metabolism and thus the effects of Se on various downstream functions: an effect that would be exacerbated if Se intake is low. Future research should investigate this relationship.

\section{Conclusions}

A better understanding of the immune and inflammatory responses that occur during COVID-19 is essential to develop adequate therapeutic approaches. Clearly, there are many factors that influence the response to SARS-CoV-2 infection and the subsequent disease outcomes. Given the crucial role of nutrition, and in particular micronutrients such as Se, in the immune and inflammatory responses ${ }^{(88,108-110)}$, it is also fundamental to understand how nutritional status could influence the ability to respond to the SARS-CoV-2. The significant body of evidence from human, animal and cell culture studies indicates that Se status is an important factor in response to viral infections, notably RNA viral infections and including respiratory virus infections. Understanding of these effects is strengthened by knowledge of the underlying biochemical mechanisms discussed in this review and together indicate that Se status may also influence human response to the SARS-CoV2 infection. The one limited study to date suggests that Se status is indeed associated with COVID-19 disease outcome ${ }^{(85)}$ and, therefore, we propose that Se status is an additional risk factor that should be considered as a determinant of SARS-CoV2 infection outcome, particularly in populations where Se intake is sub-optimal or low. Susceptibility to SARS-CoV2 infection is dependent on many factors such as age, BMI, obesity, type 2 diabetes and cardiovascular dysfunction, and in this context of COVID-19 progression being multifactorial, the relative importance of Se status remains to be determined. However, we suggest that the use of appropriate markers such as erythrocyte $\mathrm{Se}^{(83)}$ to monitor Se status of COVID-19 patients and possible supplementation may be beneficial in limiting the severity of symptoms, and secondly that authorities in countries where Se status is regarded as suboptimal may consider Se supplementation as a public health strategy. Finally, on the basis of findings with other RNA viruses, it will be important to assess the extent to which Se status affects the mutation rate and pathogenicity of SARS-CoV-2.

\section{Acknowledgements}

The authors thank Robert Gordon University and Newcastle University for supporting this work.

No financial support was received for this work.

G. B., C. M., D. K. M. and J. E. H. contributed to the conception and design of this review, drafting and revising of the article. G. B., C. M., D. K. M. and J. E. H. approved the final version.

The authors declare that there are no conflicts of interest.

\section{References}

1. Loscalzo J (2014) Keshan disease, selenium deficiency, and the selenoproteome. $N$ Engl J Med 370, 1756-1760.

2. Rayman MP (2000) The importance of selenium to human health. Lancet 356, 233-241.

3. Guillin OM, Vindry C, Ohlmann T, et al. (2019) Selenium, selenoproteins and viral infection. Nutrients 11, 2101.

4. Huang C, Wang Y, Li X, et al. (2020) Clinical features of patients infected with 2019 novel coronavirus in Wuhan, China. Lancet 395, 497-506.

5. Rayman MP \& Rayman MP (2002) The argument for increasing selenium intake. Proc Nutr Soc 61, 203-215.

6. Johnson CC, Fordyce FM \& Rayman MP (2010) Symposium on 'Geographical and geological influences on nutrition': factors controlling the distribution of selenium in the environment and their impact on health and nutrition. Proc Nutr Soc 69, 119-132.

7. Almeida JD, Berry DM, Cunningham CH, et al. (1968) Virology: coronaviruses. Nature $\mathbf{2 2 0}, 650$.

8. Coronaviridae Study Group of the International Committee on Taxonomy of Viruses (2020) The species severe acute respiratory syndrome-related coronavirus: classifying 2019-nCoV and naming it SARS-CoV-2. Nat Microbiol 5, 536-544.

9. Tay MZ, Poh CM, Renia L, et al. (2020) The trinity of COVID-19: immunity, inflammation and intervention. Nat Rev Immunol 20, 363-374.

10. Xu H, Zhong L, Deng J, et al. (2020) High expression of ACE2 receptor of 2019-nCoV on the epithelial cells of oral mucosa. Int J Oral Sci 12, 8-12.

11. Chen N, Zhou M, Dong X, et al. (2020) Epidemiological and clinical characteristics of 99 cases of 2019 novel coronavirus pneumonia in Wuhan, China: a descriptive study. Lancet 395, 507-513.

12. Chu KH, Tsang WK, Tang CS, et al. (2005) Acute renal impairment in coronavirus-associated severe acute respiratory syndrome. Kidney Int 67, 698-705.

13. Ruan Q, Yang K, Wang W, et al. (2020) Clinical predictors of mortality due to COVID-19 based on an analysis of data of 150 patients from Wuhan, China. Intensive Care Med 46, 846-848. 
14. Beck MA, Levander OA \& Handy J (2003) Selenium deficiency and viral infection. J Nutr 133, 1463S-1467S.

15. Beck MA, Shi Q, Morris VC, et al. (1995) Rapid genomic evolution of a non-virulent coxsackievirus B3 in seleniumdeficient mice results in selection of identical virulent isolates. Nat Med 1, 433-436.

16. Beck MA (1997) Rapid genomic evolution of a non-virulent coxsackievirus B3 in selenium-deficient mice. Biomed Environ Sci 10, 307-315.

17. Beck MA, Nelson HK, Shi Q, et al. (2001) Selenium deficiency increases the pathology of an influenza virus infection. FASEBJ 15, 1481-1483.

18. Jaspers I, Zhang W, Brighton LE, et al. (2007) Selenium deficiency alters epithelial cell morphology and responses to influenza. Free Radic Biol Med 42, 1826-1837.

19. Nelson HK, Shi Q, Van Dael P, et al. (2001) Host nutritional selenium status as a driving force for influenza virus mutations. FASEB J 15, 1846-1848.

20. Look MP, Rockstroh JK, Rao GS, et al. (1997) Serum selenium versus lymphocyte subsets and markers of disease progression and inflammatory response in human immunodeficiency virus-1 infection. Biol Trace Elem Res 56, 31-41.

21. Baum MK, Shor-Posner G, Lai S, et al. (1997) High risk of HIV-related mortality is associated with selenium deficiency. J Acquir Immune Defic Syndr Hum Retrovirol 15, 370-374.

22. Fang LQ, Goeijenbier M, Zuo SQ, et al. (2015) The association between hantavirus infection and selenium deficiency in mainland China. Viruses 7, 333-351.

23. Verma S, Molina Y, Lo YY, et al. (2008) In vitro effects of selenium deficiency on West Nile virus replication and cytopathogenicity. Virol J 5, 66

24. Broome CS, McArdle F, Kyle JA, et al. (2004) An increase in selenium intake improves immune function and poliovirus handling in adults with marginal selenium status. Am J Clin Nutr 80, 154-162.

25. Hesketh J (2008) Nutrigenomics and selenium: gene expression patterns, physiological targets, and genetics. Annu Rev Nutr 28, 157-177.

26. Meplan C (2015) Selenium and chronic diseases: a nutritional genomics perspective. Nutrients 7, 3621-3651.

27. Bellinger FP, Raman AV, Reeves MA, et al. (2009) Regulation and function of selenoproteins in human disease. Biochem $J$ 422, 11-22.

28. Kipp A, Banning A, van Schothorst EM, et al. (2009) Four selenoproteins, protein biosynthesis, and Wnt signalling are particularly sensitive to limited selenium intake in mouse colon. Mol Nutr Food Res 53, 1561-1572.

29. Kipp AP, Banning A, van Schothorst EM, et al. (2012) Marginal selenium deficiency down-regulates inflammation-related genes in splenic leukocytes of the mouse. JNutrBiochem 23, 1170-1177.

30. Meplan C, Johnson IT, Polley AC, et al. (2016) Transcriptomics and proteomics show that selenium affects inflammation, cytoskeleton, and cancer pathways in human rectal biopsies. FASEB J 30, 2812-2825.

31. Khomich OA, Kochetkov SN, Bartosch B, et al. (2018) Redox Biology of Respiratory Viral Infections. Viruses 10, 392.

32. Chen X, Ren F, Hesketh J, et al. (2012) Selenium blocks porcine circovirus type 2 replication promotion induced by oxidative stress by improving GPx1 expression. Free Radic Biol Med 53, 395-405.

33. Beck MA, Esworthy RS, Ho YS, et al. (1998) Glutathione peroxidase protects mice from viral-induced myocarditis. FASEBJ 12, 1143-1149.

34. Yatmaz S, Seow HJ, Gualano RC, et al. (2013) Glutathione peroxidase-1 reduces influenza A virus-induced lung inflammation. Am J Respir Cell Mol Biol 48, 17-26.
35. Sheridan PA, Zhong N, Carlson BA, et al. (2007) Decreased selenoprotein expression alters the immune response during influenza virus infection in mice. J Nutr 137, 1466-1471.

36. Pohanka M (2013) Role of oxidative stress in infectious diseases. A review. Folia Microbiol (Praha) 58, 503-513.

37. Buffinton GD, Christen S, Peterhans E, et al. (1992) Oxidative stress in lungs of mice infected with influenza A virus. Free Radic Res Commun 16, 99-110.

38. Hennet T, Peterhans E \& Stocker R (1992) Alterations in antioxidant defences in lung and liver of mice infected with influenza A virus. J Gen Virol 73, 39-46.

39. Amatore D, Sgarbanti R, Aquilano K, et al. (2015) Influenza virus replication in lung epithelial cells depends on redoxsensitive pathways activated by NOX4-derived ROS. Cell Microbiol 17, 131-145.

40. Amini P, Kolivand S, Saffar H, et al. (2019) Protective effect of selenium-L-methionine on radiation-induced acute pneumonitis and lung fibrosis in Rat. Curr Clin Pharmacol 14, 157164.

41. Brigelius-Flohe R, Banning A \& Schnurr K (2003) Seleniumdependent enzymes in endothelial cell function. Antioxid Redox Signal 5, 205-215.

42. Varga Z, Flammer AJ, Steiger P, et al. (2020) Endothelial cell infection and endotheliitis in COVID-19. Lancet 395, $1417-1418$.

43. Kose SA \& Naziroglu M (2014) Selenium reduces oxidative stress and calcium entry through TRPV1 channels in the neutrophils of patients with polycystic ovary syndrome. Biol Trace Elem Res 158, 136-142.

44. Kim S, Kim M, Park DY, et al. (2015) Mitochondrial reactive oxygen species modulate innate immune response to influenza A virus in human nasal epithelium. Antivir Res 119, 78-83.

45. Shi CS, Qi HY, Boularan C, et al. (2014) SARS-coronavirus open reading frame-9b suppresses innate immunity by targeting mitochondria and the MAVS/TRAF3/TRAF6 signalosome. J Immunol 193, 3080-3089.

46. Handy DE, Lubos E, Yang Y, et al. (2009) Glutathione peroxidase-1 regulates mitochondrial function to modulate redoxdependent cellular responses. J Biol Chem 284, 11913-11921.

47. Cole-Ezea P, Swan D, Shanley D, et al. (2012) Glutathione peroxidase 4 has a major role in protecting mitochondria from oxidative damage and maintaining oxidative phosphorylation complexes in gut epithelial cells. Free Radic Biol Med 53, 488-497.

48. Fung TS, Huang M \& Liu DX (2014) Coronavirus-induced ER stress response and its involvement in regulation of coronavirus-host interactions. Virus Res 194, 110-123.

49. Addinsall AB, Wright CR, Andrikopoulos S, et al. (2018) Emerging roles of endoplasmic reticulum-resident selenoproteins in the regulation of cellular stress responses and the implications for metabolic disease. Biochem $J \mathbf{4 7 5}$, 1037-1057.

50. Namgaladze D, Khodzhaeva V \& Brune B (2019) ER-mitochondria communication in cells of the innate immune system. Cells 8, 1088.

51. Kelly E, Greene CM, Carroll TP, et al. (2009) Selenoprotein S/SEPS1 modifies endoplasmic reticulum stress in $\mathrm{Z}$ variant alpha1-antitrypsin deficiency. J Biol Chem 284, 16891-16897.

52. Ye Y, Fu F, Li X, et al. (2016) Selenoprotein S is highly expressed in the blood vessels and prevents vascular smooth muscle cells from apoptosis. J Cell Biochem 117, 106-117.

53. Verma S, Hoffmann FW, Kumar M, et al. (2011) Selenoprotein $\mathrm{K}$ knockout mice exhibit deficient calcium flux in immune cells and impaired immune responses. J Immunol 186, 2127-2137. 
54. Goldson AJ, Fairweather-Tait SJ, Armah CN, et al. (2011) Effects of selenium supplementation on selenoprotein gene expression and response to influenza vaccine challenge: a randomised controlled trial. PLOS ONE 6, e14771.

55. Minakshi R, Padhan K, Rani M, et al. (2009) The SARS coronavirus $3 \mathrm{a}$ protein causes endoplasmic reticulum stress and induces ligand-independent downregulation of the type 1 interferon receptor. PLOS ONE $\mathbf{4}$, e8342.

56. Hasnain SZ, Lourie R, Das I, et al. (2012) The interplay between endoplasmic reticulum stress and inflammation. Immunol Cell Biol 90, 260-270.

57. Kaminska B (2005) MAPK signalling pathways as molecular targets for anti-inflammatory therapy - from molecular mechanisms to therapeutic benefits. Biochim Biophys Acta 1754 253-262.

58. Girard S, Kadhim H, Roy M, et al. (2009) Role of perinatal inflammation in cerebral palsy. Pediatr Neurol 40, 168-174.

59. Hendrayani SF, Al-Harbi B, Al-Ansari MM, et al. (2016) The inflammatory/cancer-related IL-6/STAT3/NF-kappaB positive feedback loop includes AUF1 and maintains the active state of breast myofibroblasts. Oncotarget 7, 41974-41985.

60. Kyriakis JM \& Avruch J (2001) Mammalian mitogen-activated protein kinase signal transduction pathways activated by stress and inflammation. Physiol Rev 81, 807-869.

61. Singh K, Chen YC, Judy JT, et al. (2020) Network analysis and transcriptome profiling identify autophagic and mitochondrial dysfunctions in SARS-CoV-2. Infection. https://doi.org/10. 1101/2020.05.13.092536.

62. Wong CK, Lam CW, Wu AK, et al. (2004) Plasma inflammatory cytokines and chemokines in severe acute respiratory syndrome. Clin Exp Immunol 136, 95-103.

63. Yoshikawa T, Hill T, Li K, et al. (2009) Severe acute respiratory syndrome (SARS) coronavirus-induced lung epithelial cytokines exacerbate SARS pathogenesis by modulating intrinsic functions of monocyte-derived macrophages and dendritic cells. J Virol 83, 3039-3048.

64. Fu Y, Cheng Y \& Wu Y (2020) Understanding SARS-CoV-2mediated inflammatory responses: from mechanisms to potential therapeutic tools. Virol Sin 35, 266-271

65. Kosmider B, Messier EM, Janssen WJ, et al. (2012) Nrf2 protects human alveolar epithelial cells against injury induced by influenza A virus. Respir Res 13, 43-43.

66. Yamada Y, Limmon GV, Zheng D, et al. (2012) Major shifts in the spatio-temporal distribution of lung antioxidant enzymes during influenza pneumonia. PLOS ONE 7, e31494.

67. Brigelius-Flohe R \& Kipp AP (2013) Selenium in the redox regulation of the Nrf2 and the Wnt pathway. Methods Enzymol 527, 65-86.

68. Tindell R, Wall SB, Li Q, et al. (2018) Selenium supplementation of lung epithelial cells enhances nuclear factor E2-related factor 2 (Nrf2) activation following thioredoxin reductase inhibition. Redox Biol 19, 331-338.

69. Tsuji PA, Carlson BA, Anderson CB, et al. (2015) Dietary selenium levels affect selenoprotein expression and support the interferon-gamma and IL-6 immune response pathways in mice. Nutrients 7, 6529-6549.

70. Berg BM, Godbout JP, Chen J, et al. (2005) Alpha-tocopherol and selenium facilitate recovery from lipopolysaccharideinduced sickness in aged mice. J Nutr 135, 1157-1163.

71. Walston J, Xue Q, Semba RD, et al. (2006) Serum antioxidants, inflammation, and total mortality in older women. Am J Epidemiol 163, 18-26.

72. Mattmiller SA, Carlson BA \& Sordillo LM (2013) Regulation of inflammation by selenium and selenoproteins: impact on eicosanoid biosynthesis. J Nutr Sci 2, e28.
73. Hu Y, McIntosh GH, Le Leu RK, et al. (2016) Supplementation with Brazil nuts and green tea extract regulates targeted biomarkers related to colorectal cancer risk in humans. Br J Nutr 116, 1901-1911.

74. Narayan V, Ravindra KC, Liao C, et al. (2015) Epigenetic regulation of inflammatory gene expression in macrophages by selenium. J Nutr Biochem 26, 138-145.

75. Shi Y, Wang Y, Shao C, et al. (2020) COVID-19 infection: the perspectives on immune responses. Cell Death Differ 27, 1451-1454.

76. Huang Z, Rose AH \& Hoffmann PR (2012) The role of selenium in inflammation and immunity: from molecular mechanisms to therapeutic opportunities. Antioxid Redox Signal 16, 705-743.

77. Maggini S, Pierre A \& Calder PC (2018) Immune function and micronutrient requirements change over the life course. Nutrients 10, 1531.

78. Ivory K, Prieto E, Spinks C, et al. (2017) Selenium supplementation has beneficial and detrimental effects on immunity to influenza vaccine in older adults. Clin Nutr 36, 407-415.

79. Steinbrenner H, Al-Quraishy S, Dkhil MA, et al. (2015) Dietary selenium in adjuvant therapy of viral and bacterial infections. Adv Nutr 6, 73-82.

80. Lee BC, Peterfi Z, Hoffmann FW, et al. (2013) MsrB1 and MICALs regulate actin assembly and macrophage function via reversible stereoselective methionine oxidation. Mol Cell 51, 397-404.

81. Lee BC, Lee SG, Choo MK, et al. (2017) Selenoprotein MsrB1 promotes anti-inflammatory cytokine gene expression in macrophages and controls immune response in vivo. Sci Rep $\mathbf{7}$, 5119 .

82. Hesse-Bahr K, Dreher I \& Kohrle J (2000) The influence of the cytokines IL-1beta and INFgamma on the expression of selenoproteins in the human hepatocarcinoma cell line HepG2. Biofactors 11, 83-85.

83. Stefanowicz F, Gashut RA, Talwar D, et al. (2014) Assessment of plasma and red cell trace element concentrations, disease severity, and outcome in patients with critical illness. J Crit Care 29, 214-218.

84. Li S, Banuelos GS, Wu L, et al. (2014) The changing selenium nutritional status of Chinese residents. Nutrients $\mathbf{6}, 1103-1114$.

85. Zhang J, Taylor EW, Bennett K, et al. (2020) Association between regional selenium status and reported outcome of COVID-19 cases in China. Am J Clin Nutr 111, 1297-1299.

86. Barazzoni R, Bischoff SC, Breda J, et al. (2020) ESPEN expert statements and practical guidance for nutritional management of individuals with SARS-CoV-2 infection. Clin Nutr 39, 1631-1638.

87. Semba RD \& Tang AM (1999) Micronutrients and the pathogenesis of human immunodeficiency virus infection. $\mathrm{Br} \mathrm{J}$ Nutr 81, 181-189.

88. Zhang L \& Liu Y (2020) Potential interventions for novel coronavirus in China: a systematic review. J Med Virol 92, 479-490.

89. Kassir R (2020) Risk of COVID-19 for patients with obesity. Obes Rev 21, e13034.

90. Peng YD, Meng K, Guan HQ, et al. (2020) Clinical characteristics and outcomes of 112 cardiovascular disease patients infected by 2019-nCoV. Zhonghua Xin Xue Guan Bing Za Zhi 48, E004.

91. Li MY, Li L, Zhang Y, et al. (2020) Expression of the SARS$\mathrm{CoV}-2$ cell receptor gene ACE2 in a wide variety of human tissues. Infect Dis Poverty 9, 45-52.

92. Pinheiro TA, Barcala-Jorge AS, Andrade JMO, et al. (2017) Obesity and malnutrition similarly alter the renin-angiotensin 
system and inflammation in mice and human adipose. J Nutr Biochem 48, 74-82.

93. Milner JJ, Rebeles J, Dhungana S, et al. (2015) Obesity increases mortality and modulates the lung metabolome during pandemic H1N1 influenza virus infection in mice. J Immunol 194, 4846-4859.

94. Maier HE, Lopez R, Sanchez N, et al. (2018) Obesity increases the duration of influenza a virus shedding in adults. J Infect Dis 218, 1378-1382

95. Patel AB \& Verma A (2020) COVID-19 and angiotensinconverting enzyme inhibitors and angiotensin receptor blockers: what is the evidence? JAMA 323, 1769-1770.

96. Tinkov AA, Ajsuvakova OP, Filippini T, et al. (2020) Selenium and selenoproteins in adipose tissue physiology and obesity. Biomolecules 10, 658

97. Azad GK \& Tomar RS (2014) Ebselen, a promising antioxidant drug: mechanisms of action and targets of biological pathways. Mol Biol Rep 41, 4865-4879.

98. Jin Z, Du X, Xu Y, et al. (2020) Structure of Mpro from SARS$\mathrm{CoV}-2$ and discovery of its inhibitors. Nature (London) $\mathbf{5 8 2}$ 289-293.

99. Sies H \& Parnham MJ (2020) Potential therapeutic use of ebselen for COVID-19 and other respiratory viral infections. Free Radic Biol Med 156, 107-112.

100. Kil J, Lobarinas E, Spankovich C, et al. (2017) Safety and efficacy of ebselen for the prevention of noise-induced hearing loss: a randomised, double-blind, placebo-controlled, phase 2 trial. Lancet 390, 969-979.

101. Masaki C, Sharpley AL, Cooper CM, et al. (2016) Effects of the potential lithium-mimetic, ebselen, on impulsivity and emotional processing. Psychopharmacology (Berl) 233, $2655-2661$.
102. Curran JE, Jowett JB, Elliott KS, et al. (2005) Genetic variation in selenoprotein $\mathrm{S}$ influences inflammatory response. Nat Genet 37, 1234-1241

103. Crosley LK, Bashir S, Nicol F, et al. (2013) The singlenucleotide polymorphism (GPX4c718t) in the glutathione peroxidase 4 gene influences endothelial cell function: interaction with selenium and fatty acids. Mol Nutr Food Res 57, 2185-2194.

104. Nogales A \& DeDiego M (2019) Host single nucleotide polymorphisms modulating influenza A virus disease in humans. Pathogens 8, 168.

105. Wada M, Lokugamage KG, Nakagawa K, et al. (2018) Interplay between coronavirus, a cytoplasmic RNA virus, and nonsense-mediated mRNA decay pathway. Proc Natl Acad Sci U S A 115, E10157-E10166.

106. Seyedali A \& Berry MJ (2014) Nonsense-mediated decay factors are involved in the regulation of selenoprotein mRNA levels during selenium deficiency. RNA 20, 1248-1256.

107. Zupanic A, Meplan C, Huguenin GV, et al. (2016) Modeling and gene knockdown to assess the contribution of nonsense-mediated decay, premature termination, and selenocysteine insertion to the selenoprotein hierarchy. RNA 22, 1076-1084.

108. Avery JC \& Hoffmann PR (2018) Selenium, selenoproteins, and immunity. Nutrients 10, 1203.

109. Calder PC, Carr AC, Gombart AF, et al. (2020) Optimal nutritional status for a well-functioning immune system is an important factor to protect against viral infections. Nutrients 12, 1181.

110. Gombart AF, Pierre A \& Maggini S (2020) A review of micronutrients and the immune system-working in harmony to reduce the risk of infection. Nutrients $\mathbf{1 2}, 236$ 